\title{
El Art Thinking como catalizador del pensamiento divergente
}

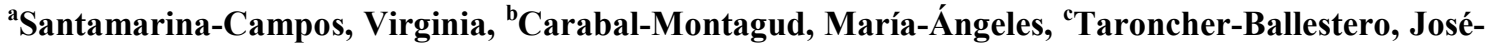 Manuel, ${ }^{\mathrm{d}}$ Moreno-Ribelles, Enrique}

${ }^{a-b}$ Departamento de Conservación y Restauración de Bienes Culturales de la Universitat Politècnica de València, ${ }^{\mathrm{c}}$ Conselleria de Educación, Cultura y Deporte y ${ }^{\mathrm{d}}$ Departamento de Comunicación Audiovisual, Documentación e Historia del Arte, Universitat Politècnica de València.

\begin{abstract}
Practical training in the university environment, without attention to creativity, design and innovative thinking, is ineffective. For this reason we have tried to find a new learning model that combines STEM areas with artistic disciplines. So that the student through the exercise of design, enhances their flexible thinking and creativity, essential ingredients when it comes to innovating.

The use of tools such as Art Thinking and the search for new learning spaces, have allowed us to create a favorable climate for the development of creativity and discover that the combination of technical training with skills associated with the artistic field, are positioned as the way ideal for training the professionals of the future.

Thus, within the strategy of achieving professional competencies, it has been planned to work together and collaboratively between the creative, technological and management disciplines that coexist on the campus of the Polytechnic University of Valencia, in the implementation of new training spaces, that favor the acquisition of transversal competences and enhance the teaching and learning process through visible and responsible learning and under STEAM strategies.
\end{abstract}

Keywords: Art Thinking, STEAM, professional skills, transversal skills, visible learning, responsible learning, Sustainable Development Goals, SDGs

\section{Resumen}

La formación práctica en el ámbito universitario, sin atención a la creatividad, el diseño y el pensamiento innovador, resulta poco efectiva. Por dicho motivo hemos tratado de buscar un nuevo modelo de aprendizaje, que combine las áreas STEM con las disciplinas artísticas. Para que el alumno a través del ejercicio del diseño, potencie su pensamiento flexible y creatividad, ingredientes indispensables cuando se trata de innovar.

El empleo de herramientas como el Art Thinking y la búsqueda de nuevos espacios de aprendizaje, nos han permito crear un clima propicio para el desarrollo de la creatividad y descubrir que la combinación de formación técnica con habilidades asociadas al campo artístico, se posicionan como el camino ideal para formar a los profesionales del futuro.

De este modo, dentro de la estrategia de alcanzar las competencias profesionales se ha previsto trabajar de forma conjunta y colaborativa entre las disciplinas creativas, tecnológicas y de gestión que conviven en el campus de la Universidad Politécnica de Valencia, en la implementación de nuevos espacios formativos, que favorezcan la adquisición de las competencias 
transversales y potencien el proceso de enseñanza y aprendizaje a través de un aprendizaje visible y responsable y bajo estrategias STEAM.

Palabras clave: Art Thinking, STEAM, competencias profesionales, competencias transversales, aprendizaje visible, aprendizaje responsable, Objetivos de Desarrollo Sostenible, ODS.

\section{Introducción}

La formación práctica en el ámbito universitario, sin atención a la creatividad, el diseño y el pensamiento innovador, resulta poco efectiva. Como indicaba Marcel Just, director del Centro de Imágenes Cerebrales Cognitivas en la Universidad Carnegie Mellon, fuimos biológicamente diseñados para reaccionar ante estímulos visuales y comunicarnos a través de ellos. Es por ello, por lo que muchas veces nos llama más la atención imágenes en lugar de un largo texto (Just \& Ludtke, 2010). Por dicho motivo hemos tratado de buscar un nuevo modelo de aprendizaje, que combine las áreas STEM con las disciplinas artísticas. Para que el alumno a través del ejercicio del diseño, potencie su pensamiento flexible y creatividad, ingredientes indispensables cuando se trata de innovar.

El empleo de herramientas como el Art Thinking y la búsqueda de nuevos espacios de aprendizaje, en ocasiones fuera del aula y en otras transformando el propio espacio de la clase en un "rincón para soñar", nos han permito crear un clima propicio para el desarrollo de la creatividad y descubrir que la combinación de formación técnica con habilidades asociadas al campo artístico, se posicionan como el camino ideal para formar a los profesionales del futuro.

De este modo, dentro de la estrategia de alcanzar las competencias profesionales se ha previsto trabajar de forma conjunta y colaborativa entre las disciplinas creativas, tecnológicas y de gestión que conviven en el campus de la Universidad Politécnica de Valencia, en la implementación de nuevos espacios formativos, que favorezcan la adquisición de las competencias transversales y potencien el proceso de enseñanza y aprendizaje a través de:

1. Un APRENDIZAJE VISIBLE a través del diseño (mapas conceptuales colaborativos), donde alumnos y profesores se convierten en productores de contenidos, propiciando el pensamiento divergente.

2. Un APRENDIZAJE RESPONSABLE, a través de la incorporación de los ODS (Objetivos de Desarrollo Sostenible) en las asignaturas implicadas, fomentando la sensibilización con los problemas del desarrollo y de la sostenibilidad ambiental, como actuales y futuros ejecutores y responsables de implementar estos objetivos.

3. Un APRENDIZAJE en STEAM, a través de la combinación de las habilidades artísticas y creativas con la educación STEM, empleando la práctica como forma de adquirir conocimientos (pedagogía inversa) y potenciando el pensamiento flexible y su creatividad, ingredientes indispensables cuando se trata de innovar.

Nuestro objetivo, es aportar las herramientas necesarias para fomentar la resolución de problemas, trabajar la capacidad de análisis o aprender a trabajar en equipo, aportando a los estudiantes las soft skills tanto de perfiles técnicos como creativos en un ambiente divergente. Es decir, formar profesionales más sólidos, creativos y con una visión más amplia. Que les permita encontrar respuestas múltiples a una misma pregunta, fomentando estilos cognitivos favorecedores del desarrollo de la creatividad y ayudándoles a construir proyectos de investigación e innovación responsables que cumplan con los ODS. 
En concreto este trabajo se ha centrado en el diseño de actividades de aprendizaje vinculadas a la competencia Arts \& Design, es decir tareas enfocadas a trabajar las habilidades artísticas y creativas, que favorezcan el pensamiento divergente, para dotar a los alumnos de instrumentos que les permita encontrar soluciones inteligentes e innovadoras a problemas y retos. Por tanto, a través de estas prácticas se pretende alcanzar las siguientes competencias transversales (Universitat Politècnica de València, 2013):

Table 1. Competencias transversales vinculadas a la competencia Arts \& Design. Fuente: Elaboración propia a partir de (Universitat Politècnica de València, 2013).

\begin{tabular}{|c|c|c|c|}
\hline CT & Nivel de dominio 1 & Indicador & Indicador Arts \& Design \\
\hline $\begin{array}{l}\text { Comprensión } \\
\text { e integración }\end{array}$ & $\begin{array}{l}\text { N2. Proceder con } \\
\text { lógica y relacionar } \\
\text { e } r \text { integrar } \\
\text { conceptos } \\
\text { situaciones } \\
\text { complejas. }\end{array}$ & $\begin{array}{lr}\text { Clasificar } & \text { y } \\
\text { establecer relaciones } \\
\text { significativas, y } \\
\text { diferenciar los } \\
\text { factores causantes o } \\
\text { consecuentes de un } \\
\text { hecho. }\end{array}$ & $\begin{array}{l}\text { El aprendizaje visible a través del } \\
\text { diseño colaborativo de arboles de } \\
\text { problemas, ayuda a entender las } \\
\text { relaciones causales (¿qué sucede? } \\
\text { ¿por qué sucede?). }\end{array}$ \\
\hline $\begin{array}{l}\text { Aplicación y } \\
\text { pensamiento } \\
\text { práctico }\end{array}$ & $\begin{array}{l}\text { N3. Diseñar un } \\
\text { plan para abordar } \\
\text { situaciones } \\
\text { complejas de forma } \\
\text { individual o en } \\
\text { colaboración con } \\
\text { otros. }\end{array}$ & $\begin{array}{l}\text { Establecer/acordar } \\
\text { objetivos concretos } \\
\text { en relación con las } \\
\text { situaciones } \\
\text { complejas que se le } \\
\text { plantean. }\end{array}$ & $\begin{array}{l}\text { El aprendizaje visible a través del } \\
\text { diseño colaborativo de arboles de } \\
\text { objetivos, ayuda a entender las } \\
\text { relaciones medio-fin (¿cómo se puede } \\
\text { solucionar?). }\end{array}$ \\
\hline $\begin{array}{lr}\text { Análisis } & \text { y } \\
\text { resolución } & \text { de } \\
\text { problemas } & \end{array}$ & $\begin{array}{l}\text { N3. Solucionar } \\
\text { problemas en } \\
\text { equipo, } \\
\begin{array}{l}\text { contextos } \\
\text { diferentes y en } \\
\text { profundidad. }\end{array}\end{array}$ & $\begin{array}{l}\text { Analizar las causas y } \\
\text { efectos de los } \\
\text { problemas desde un } \\
\text { enfoque global. }\end{array}$ & $\begin{array}{l}\text { El aprendizaje visible a través del } \\
\text { diseño mapas conceptuales } \\
\text { colaborativos verticales produce el } \\
\text { mejor modelo sistémico, porque } \\
\text { sintetiza diferentes puntos de vista. }\end{array}$ \\
\hline $\begin{array}{l}\text { Diseño } \\
\text { proyecto }\end{array}$ & $\begin{array}{l}\text { N2. Planificar } \\
\text { proyectos en } \\
\text { colaboración con } \\
\text { otros }\end{array}$ & $\begin{array}{l}\text { Fundamentar la } \\
\text { necesidad del } \\
\text { proyecto, formular } \\
\text { los objetivos y } \\
\text { resultados respecto a } \\
\text { las necesidades. }\end{array}$ & $\begin{array}{l}\text { La activación del pensamiento } \\
\text { divergente a través del ejercicio de } \\
\text { diseño colaborativo permite encontrar } \\
\text { respuestas múltiples a una misma } \\
\text { pregunta, aportando soluciones } \\
\text { creativas. }\end{array}$ \\
\hline $\begin{array}{lr}\text { Trabajo } & \text { en } \\
\text { equipo } & y \\
\text { liderazgo } & \end{array}$ & $\begin{array}{lr}\text { N3. Contribuir al } \\
\text { desarrollo } & y \\
\text { consolidación } & \text { del } \\
\text { equipo } & \end{array}$ & $\begin{array}{l}\text { Comunicarse y } \\
\text { relacionarse } \\
\text { contribuyendo a la } \\
\text { cohesión del equipo }\end{array}$ & $\begin{array}{l}\text { El ejercicio de diseño colaborativo } \\
\text { produce un diseño comprensible } \\
\text { porque todos participan en la } \\
\text { construcción del modelo, generando } \\
\text { sentimiento de empoderamiento. }\end{array}$ \\
\hline Responsabilid & Mostrar & Satisfacer, mediante & La comunicación visual a través del \\
\hline
\end{tabular}

\footnotetext{
${ }^{1}$ Niveles de dominio: Nivel 1 (N1), Nivel 2 (N2) y Nivel 3 (N3), en relación con las competencias transversales de la Universitat Politècnica de València.
} 


\begin{tabular}{|c|c|c|c|}
\hline $\begin{array}{l}\text { ad ética } y \\
\text { profesional }\end{array}$ & $\begin{array}{l}\text { argumentar la } \\
\text { pertinencia de los } \\
\text { comportamientos y } \\
\text { juicios que se } \\
\text { emiten. }\end{array}$ & $\begin{array}{l}\text { el diálogo, alguna } \\
\text { necesidad vinculada } \\
\text { a la convivencia y no } \\
\text { discriminar a las } \\
\text { personas. }\end{array}$ & $\begin{array}{l}\text { dibujo permite hacer visibles nuestros } \\
\text { modelos metales, facilitando el } \\
\text { dialogo y eliminando barreras. }\end{array}$ \\
\hline $\begin{array}{l}\text { Comunicación } \\
\text { efectiva }\end{array}$ & $\begin{array}{lr}\text { N2. Ilustrar } & \text { el } \\
\text { discurso } & \text { para } \\
\text { facilitar } & \text { su } \\
\text { comprensión. } & \end{array}$ & $\begin{array}{l}\text { Reforzar ideas con la } \\
\text { ayuda de los medios } \\
\text { de apoyo. }\end{array}$ & $\begin{array}{l}\text { El Marketing Visual, a través del } \\
\text { dibujo/diseño permite comunicar de } \\
\text { forma más efectiva una idea (el } \\
\text { cerebro procesa la información visual } \\
60.000 \text { veces más rápido que el } \\
\text { texto). }\end{array}$ \\
\hline $\begin{array}{l}\text { Pensamiento } \\
\text { crítico }\end{array}$ & $\begin{array}{l}\text { N2. Analizar si } \\
\text { existe coherencia } \\
\text { entre los juicios } \\
\text { propios y ajenos, } \\
\text { valorando sus } \\
\text { implicaciones } \\
\text { prácticas. }\end{array}$ & $\begin{array}{l}\text { Valorar los juicios } \\
\text { ajenos. }\end{array}$ & $\begin{array}{l}\text { La activación del pensamiento } \\
\text { divergente a través del ejercicio de } \\
\text { dibujo aporta claridad, haciendo las } \\
\text { ideas visibles, tangibles y } \\
\text { secuenciales, facilitando el análisis de } \\
\text { los diferentes puntos de vista. }\end{array}$ \\
\hline $\begin{array}{l}\text { Aprendizaje } \\
\text { permanente }\end{array}$ & $\begin{array}{l}\text { N3. Desarrollar } \\
\text { estrategias de } \\
\text { aprendizaje propias }\end{array}$ & $\begin{array}{l}\text { Diseñar un proceso } \\
\text { de aprendizaje para } \\
\text { lograr sus objetivos }\end{array}$ & $\begin{array}{l}\text { El aprendizaje visible convierte al } \\
\text { alumno en productor de contenidos, } \\
\text { propiciando el pensamiento } \\
\text { divergente. }\end{array}$ \\
\hline
\end{tabular}

En paralelo a la adquisición de las competencias transversales, las prácticas planteadas permitirán la formación en los siguientes objetivos de desarrollo sostenible (United Nations, 2015):

4.- Garantizar una educación de calidad inclusiva y equitativa, y promover las oportunidades de aprendizaje permanente para todos.

5.- Alcanzar la igualdad entre los géneros y empoderar a todas las mujeres y niñas.

8.- Fomentar el crecimiento económico sostenido, inclusivo y sostenible, el empleo pleno y productivo, y el trabajo decente para todos.

9.- Desarrollar infraestructuras resilientes, promover la industrialización inclusiva y sostenible, y fomentar la innovación.

17.- Fortalecer los medios de ejecución y reavivar la alianza mundial para el desarrollo sostenible. 


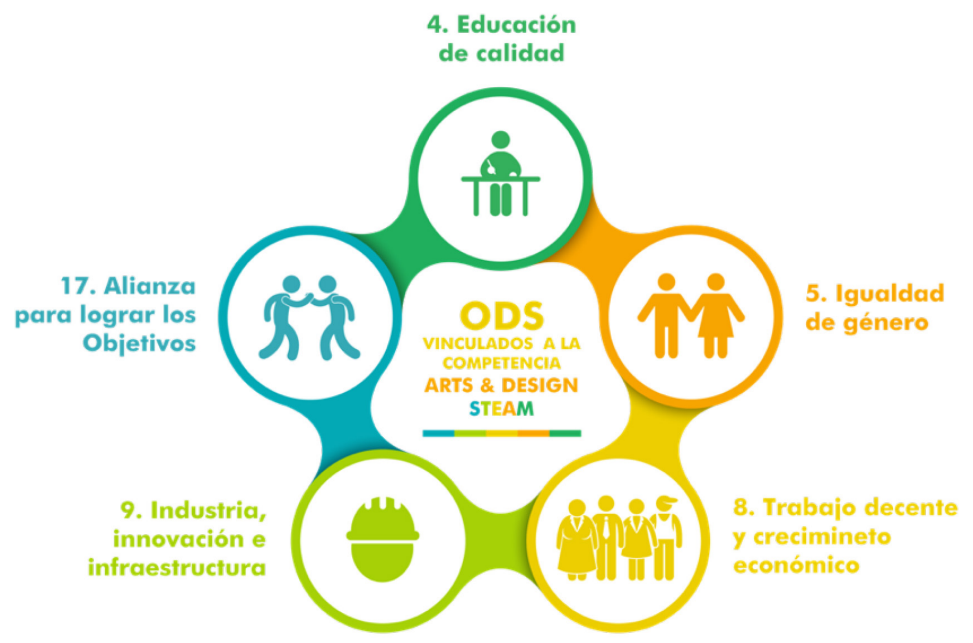

Figure 1. ODS vinculados las a las actividades de aprendizaje de la competencia Arts \& Design. Fuente: elaboración propia a partir de (United Nations, 2015).

\section{Metodología}

El objetivo del presente trabajo es diseñar y evaluar el impacto, de actividades de aprendizaje enfocadas a la adquisición de la competencia Arts \& Design, de modo que puedan ser implementadas en la educación STEM, con el objetivo de mejorar la educación científico-técnica, a través del uso del aprendizaje en competencias STEAM,

Además, las tareas de aprendizaje permitirán trabajar de forma sencilla los ODS a través de cuatro ejes: aprendizaje y enseñanza, investigación, gestión y gobernanza, y liderazgo social, descubriendo en los alumnos, la potencialidad del Arts \& Design para conseguir los ODS, fomentando la sensibilización con los problemas del desarrollo y de la sostenibilidad ambiental, como actuales y futuros ejecutores y responsables de implementar estos objetivos.

Las actividades de aprendizaje se han implementado en el primer cuatrimestre del curso 2019-2020 en asignaturas vinculadas a gestión de proyectos del Máster en Conservación y Restauración de Bienes Culturales de la Universitat Politècnica de València. Al concluir las materias se les ha enviado a los alumnos un test para evaluar la eficacia de la aplicación de la competencia.

\section{Diseño de las actividades de aprendizaje vinculadas a la competencia Arts \& Design}

\section{Práctica 1. ¿DIBUJAMOS UNA IDEA?}

La combinación de tarjetas móviles de colores (Post-it ${ }^{\circ}$ ) y el lenguaje visual para diseñar mapas conceptuales sobre grandes formatos verticales de papel o sobre la pared, supone un cambio importante en la construcción de modelos cooperativos. Por un lado, el empleo del lenguaje visual facilita el hacer las ideas visibles, tangibles y secuenciales, y fomentar el pensamiento divergente colaborativo. Por otro lado, el uso de tarjetas de colores permite atribuir categorías, caracterizar los procesos, etc., y por tanto mejorar la organización y estructuración de los mapas, ya que las reiteraciones se hacen con mayor fluidez. Para la realización de esta será necesario formar un grupo de 4 alumnos, respetando la paridad (Santamarina, Carabal, de Miguel, \& de Miguel, 2018a). 


\section{$1^{\text {a }}$ PARTE}

\begin{tabular}{|c|c|}
\hline Modo trabajo & Individual \\
\hline Material & Hoja de papel A3 y marcadores de colores. \\
\hline Descripción & $\begin{array}{l}\text { Utilizando palabras claves, representar un proceso (SENCILLO) que refleje la } \\
\text { solución del problema planteado en el aula. } \\
\text { El problema puede platearse a los alumnos a través de una imagen acompañada } \\
\text { de una pregunta que plantee un reto. } \\
\text { La solución debe de cumplir con los ODS. Se debe evitar emplear frases. Una } \\
\text { vez terminado poner boca abajo y retirarlos. Trabajar a cierta distancia para no } \\
\text { visualizar lo que realizan los demás compañeros. }\end{array}$ \\
\hline \multicolumn{2}{|l|}{$2^{\text {a PARTE }}$} \\
\hline Modo trabajo & Individual \\
\hline Material & Hoja de papel A3 y marcadores. \\
\hline Descripción & $\begin{array}{l}\text { Sin utilizar palabras, dibujar el proceso descrito en el ejercicio anterior. Una vez } \\
\text { terminado poner boca abajo y retirarlos. }\end{array}$ \\
\hline \multicolumn{2}{|l|}{ 3a PARTE } \\
\hline Modo trabajo & Individual \\
\hline Material & Hoja de papel A3, marcadores y Post-it巴 de colores y tijeras. \\
\hline Descripción & $\begin{array}{l}\text { Sin utilizar palabras, dibujar el proceso descrito en el ejercicio anterior, pero esta } \\
\text { vez utilizando tarjetas móviles de colores (Post-it } \AA) \text {, un dibujo por tarjeta, y } \\
\text { estas se van colocando y organizando en una hoja de formato A3. No se debe } \\
\text { buscar repetir el proceso anterior, es necesario olvidar lo dibujado y volver a } \\
\text { empezar. }\end{array}$ \\
\hline
\end{tabular}

Tras finalizar los dos primeros trabajos, se pegan en la pared, en la fila superior los primeros y la fila inferior los segundo, colocando en la misma columna los de cada compañero, en grupo se analizan qué cualidades tienen en común. Se lanzan las siguientes preguntas y se debate en grupo:

Pregunta 1 ¿qué tienen en común los diseños de la $1^{\mathrm{a}}, 2^{\mathrm{a}}$ y $3^{\mathrm{a}}$ parte?, ¿qué elementos contienen los diseños?.

Pregunta 2 ¿qué representan los nodos?, ¿qué representan los enlaces?.

Pregunta 3 ¿qué diferencia hay entre los diseños de la $1^{\mathrm{a}}, 2^{\mathrm{a}}$ y $3^{\mathrm{a}}$ parte?

\section{$4^{\text {a }}$ PARTE}

\begin{tabular}{|l|l|}
\hline Modo trabajo & Grupal (Paridad) \\
\hline Material & Papel continuo, marcadores y Post-it@ de colores y tijeras. \\
\hline Descripción & $\begin{array}{l}\text { Partiendo de los mapas realizados en el } 3^{\circ} \text { parte, se repite el proceso reutilizando las } \\
\text { tarjetas, pero esta vez se trabaja en grupo. Sobre el papel continuo o pared se colocan } \\
\text { los ejercicios para facilitar la reutilización de las tarjetas. }\end{array}$ \\
\hline
\end{tabular}


Tras finalizar el ejercicio, se analiza el diagrama resultante. Se lanzan las siguientes preguntas y se debate en grupo:

\section{Pregunta 4 ¿QUÉ DIFERENCIA HAY ENTRE EL 2 EJERCICIO Y EL $3^{\circ}$ ? \\ Práctica 2 y 3 . ¿DISEÑAMOS ÁRBOLES?}

La técnica de diseño del árbol de problemas y objetivos para la conceptualización de ideas innovadoras, en forma de diagramas de una situación negativa con sus causas y efectos y de las relaciones "medio-fin", es una herramienta analítica y flexible, que potencia el compromiso, la transparencia, la estructuración y la participación (Santamarina, Carabal, de Miguel, \& de Miguel, 2018b).

Estas prácticas tienen el objetivo de mostrar la capacidad de análisis que aporta la técnica del diseño del árbol de problemas y objetivos, para madurar ideas innovadoras de forma colaborativa, y construir proyectos de investigación e innovación responsables que cumplan con los ODS.

Para la realización de esta será necesario formar un grupo de entre 3 o 5 alumnos, respetando la paridad.

\section{1ªATE. PREPARACIÓN DISEÑO}

\begin{tabular}{|c|c|}
\hline Modo trabajo & Grupal (Paridad) \\
\hline Material & Papel continuo, marcadores y Post-it ${ }^{\circledR}$ de colores y tijeras. \\
\hline Descripción & $\begin{array}{l}1^{\circ} \text {. Se plantea un problema a los alumnos a través de una imagen o un texto breve } \\
\text { (máximo un folio) que describa una situación negativa a resolver. Dicha situación } \\
\text { recogerá aspectos vinculados con los ODS. } \\
2^{\circ} \text {. Se prepara papel continuo o un formato superior a A3, colocándolo preferiblemente } \\
\text { sobre la pared. } \\
3^{\text {o }} \text {. Cada participante debe tomar un paquete de Post-it }{ }^{\circledR} \text {, todos del mismo color. En } \\
\text { cada tarjeta, individualmente se ira escribiendo o dibujando un problema relacionado } \\
\text { con la necesidad a resolver. Es muy importante escribir/dibujar un único problema por } \\
\text { tarjeta, y emplear el mínimo número de palabras posibles o dibujos sencillos. Las } \\
\text { tarjetas se van pegando en el papel, sin seguir ningún orden especifico. Es importante } \\
\text { que esta primera tarea se realice de forma individual, sin realizar comentarios entre el } \\
\text { equipo. } \\
4^{\circ} \text {. Identificados todos los problemas de forma individual se realiza una puesta en } \\
\text { común, en la que se exponen y debaten, de forma respetuosa los problemas } \\
\text { identificados por cada uno. En caso de coincidir en la identificación de problemas se } \\
\text { irán eliminando las tarjetas repetidas. }\end{array}$ \\
\hline
\end{tabular}

\section{2 a PARTE. ÁRBOL DE PROBLEMAS}

\begin{tabular}{|l|l|}
\hline Modo trabajo & Grupal (Paridad) \\
\hline Material & Papel continuo, marcadores y Post-it $®$ de colores y tijeras. \\
\hline Descripción & $\begin{array}{l}\text { Una vez todos conozcan la percepción del problema de cada uno, se procederá a } \\
\text { ordenar todas las necesidades detectadas en un árbol de problemas, partiendo de la } \\
\text { identificación del problema focal y ordenando el resto de los problemas según su } \\
\text { relación causa y efecto. Para la organización de los problemas se podrán utilizarse } \\
\text { diferentes colores de Post-it@, para jerarquizar y organizar el árbol. Para construir el }\end{array}$ \\
\hline
\end{tabular}


árbol de problemas se procederá de la siguiente manera:

$1^{\circ}$. En primer lugar, se identificarán los principales problemas existentes, y no posibles, imaginados o futuros. Hay que evitar formulaciones como la ausencia de una solución y caracterizarlos como un estado negativo existente.

$2^{\circ}$. En segundo lugar, se deberá de seleccionar y consensuar un problema focal entre todos los identificados. El problema focal debe describir de forma clara la esencia de una situación general que se considera insatisfactoria. Para decidir cuál es el problema central entre aquellos seleccionados como posibles focales, se debe realizar una breve consideración y tratar de llegar a un acuerdo. Si no existe consenso, se podrá intentar ordenar todos los problemas centrales propuestos hacia arriba y abajo según las causas y efectos, para tratar de identificar el focal. En caso de no ser posible, se deberá de adoptar temporalmente uno o varios problemas centrales. Es importante recordar que NO siempre el problema central se convierte automáticamente en objetivo principal del proyecto.

$3^{\circ}$. En tercer lugar, se desarrollará el árbol de problemas. Esta tarea consiste en identificar las causas y efectos sustanciales y directos del problema focal. De este modo, a partir de todos los problemas identificados, se construye un árbol que refleja las relaciones causa-efecto entre los problemas. En esta tarea será fundamental la cooperación, tanto en el diseño como en la revisión del árbol, para asegurar si es válido y completo, realizando los ajustes necesarios.

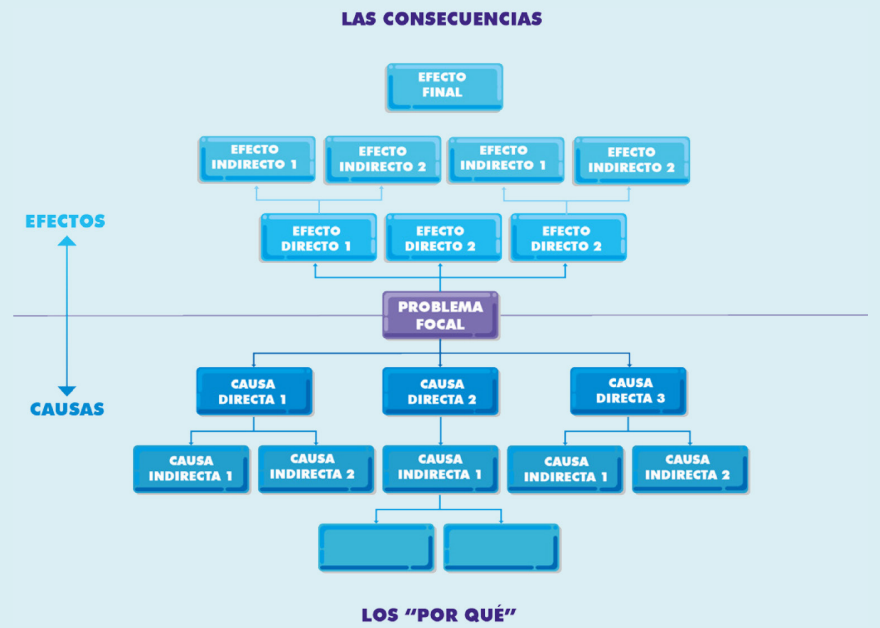

El análisis de problemas habrá permitido conocer mejor la realidad, y analizar en profundidad la idea original, buscando las debilidades y fortalezas existentes. A través de la técnica del diseño del árbol de problemas, se habrá establecido órdenes de importancia y prioridades entre las necesidades, buscando las relaciones causa-efecto entre los mismos.

Concluido el árbol de problemas, se iniciará la tercera etapa, en la que se identificarán las condiciones deseables (objetivos), identificando y visualizando las relaciones medios-fines entre los mismos y priorizando y consensuando los objetivos. 


\section{$3^{\text {a }}$ PARTE. ÁRBOL DE OBJETOS}

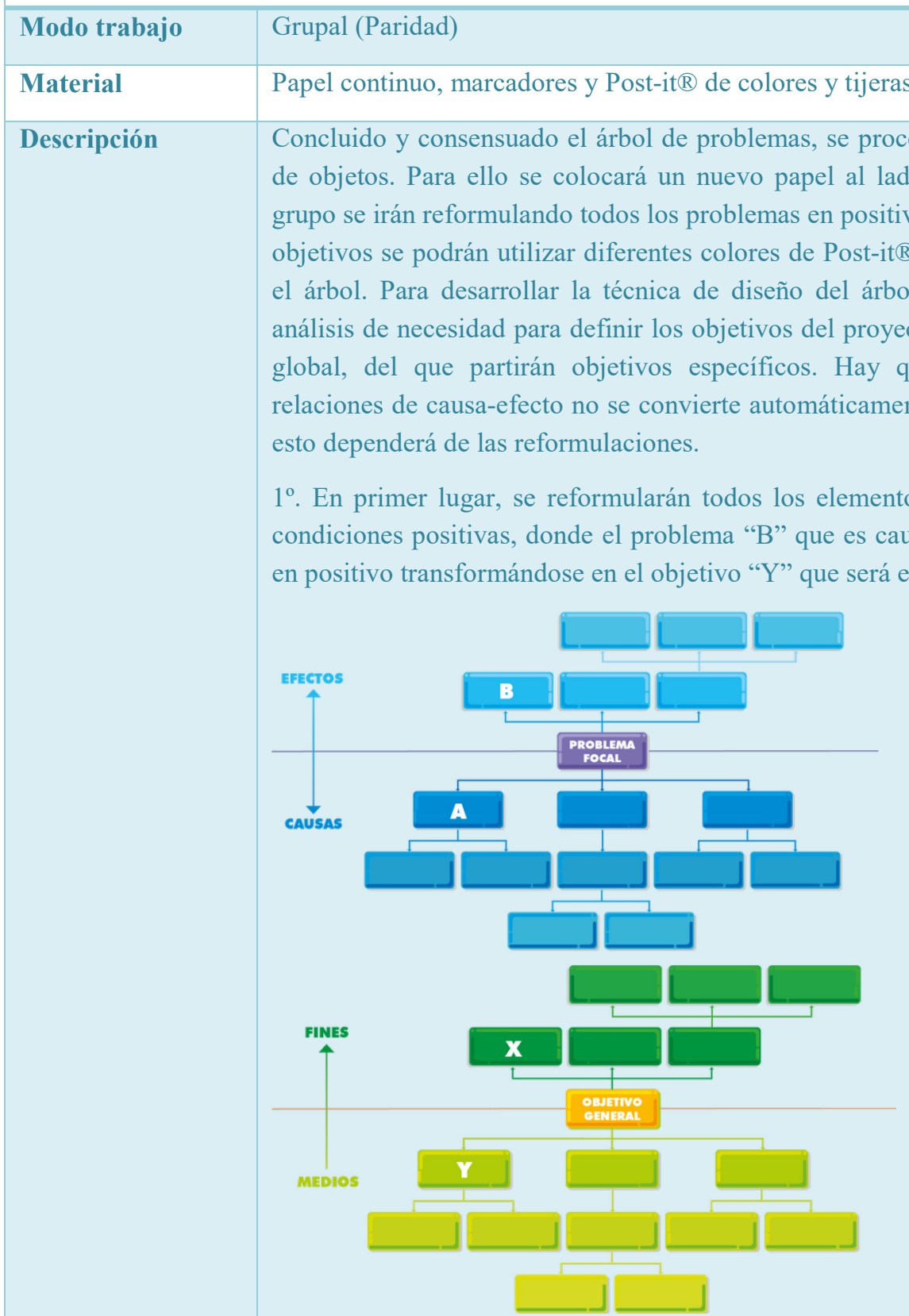

$2^{\circ}$. Una vez definidos los objetivos, es importante comprobar si los objetivos corregidos en un nivel son suficientes para lograr el objetivo del siguiente nivel.

$3^{\circ}$. Por último, una vez diseñado el árbol de objetivos, habrá que comprobar si es necesario:

Revisar los planteamientos.

Eliminar los objetivos que parecen irreales o innecesarios.

Agregar nuevos objetivos cuando sea necesario.

Trazar líneas de conexión para indicar las relaciones medios-fin. 


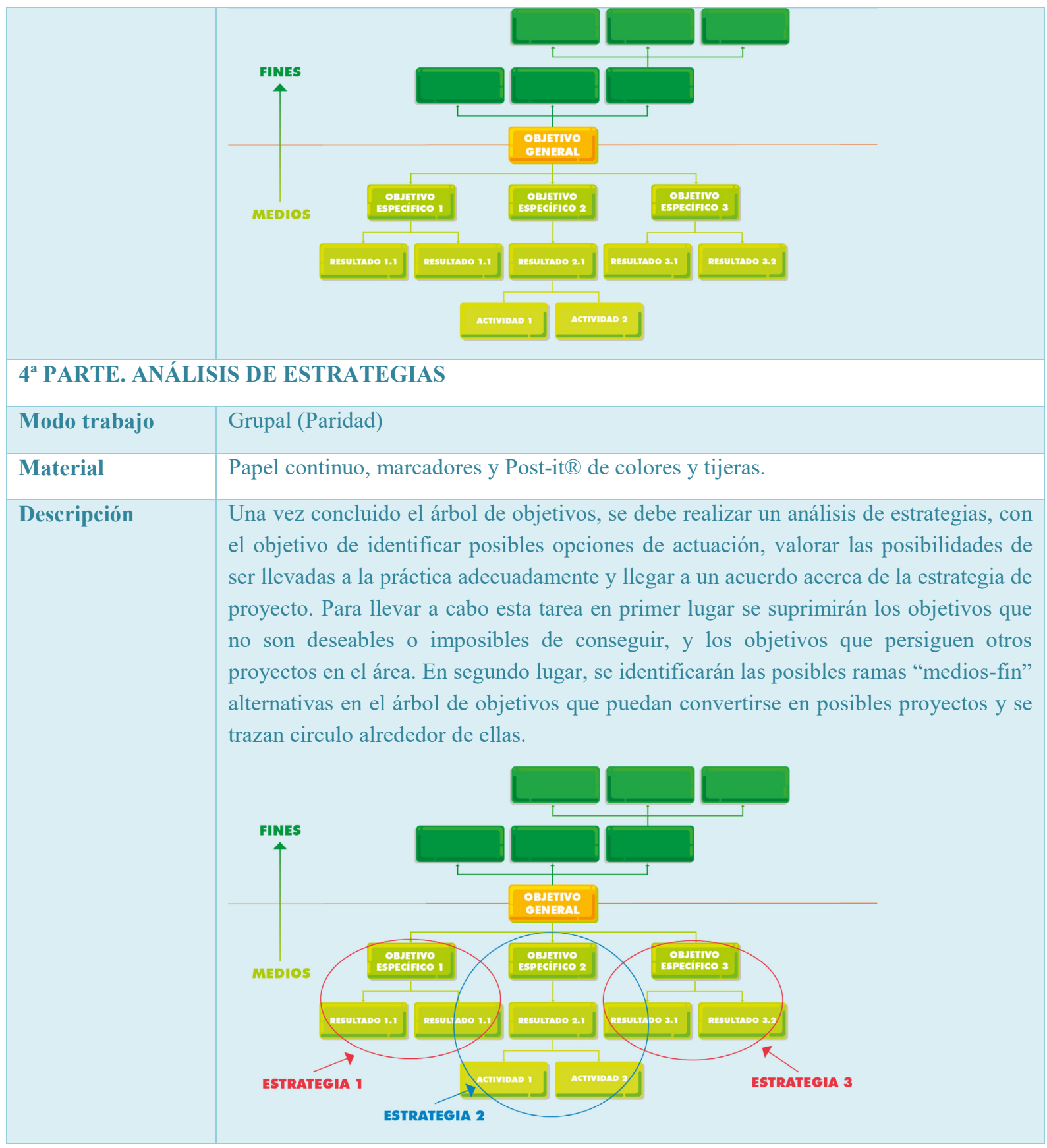

\section{Preguntas generales a los alumnos para evaluar si aplican la competencia}

Al finalizar cada cuatrimestre se les pasará un breve cuestionario para evidenciar que la estrategia que se quería enseñar ha sido interiorizada. Si los resultados son positivos, este proyecto puede ampliarse a otras asignaturas de grado o incluso a otras titulaciones que empleen la educación STEM, y todavía no hayan incorporado la competencia Arts \& Design. 
Test de evaluación de la competencia Arts \& Design:

1. ¿Dibujar y diseñar facilita encontrar soluciones a los problemas planteados?.

2. ¿Dibujar y diseñar facilita idear algo nuevo y materializarlo?.

3. Los casos reales analizados a través de practicas manipulativas y colaborativas (por ejemplo, con Post-it de colores, adhesivos de colores, formatos grandes de papel, rotuladores de colores...), ¿aportan herramientas útiles para resolver problemas?.

4. Elaborar mapas conceptuales colaborativos empleado dibujos, palabras claves y tarjeas móviles, ¿permiten encontrar respuestas múltiples a una misma pregunta?.

5. Diseñar y dibujar en grupo, ¿contribuye a la creatividad y a encontrar soluciones innovadoras?.

6. El diseño colaborativo en grupo ¿facilita sintetizar diferentes puntos de vista?.

7. Transformar el espacio del aula (modificar la distribución del mobiliario, utilizar las paredes, etc) ¿contribuye al desarrollo de la creatividad?

8. Transformar el espacio del aula (modificar la distribución del mobiliario, utilizar las paredes, etc) ¿favorece el trabajo en equipo?

\section{Resultados}

En las materias en las que se ha implementado la competencia Arts \& Design, hemos podido comprobar que ha favorecido el pensamiento divergente, logrando que los alumnos encuentren soluciones innovadoras a los problemas y retos planteados de forma más ágil. Principalmente se ha podido percibir dos cambios importantes:

$1^{\circ}$. Ha facilitado y agilizado la capacidad de análisis y la resolución de los problemas planteados en el aula, pudiendo incrementar en un $15 \%$ el numero de practicas realizadas durante el cuatrimestre. Demostrando que el potencial de los dibujos y diseños, ya que el cerebro humano puede procesar imágenes enteras que el ojo ve durante tan sólo 13 milésimas de segundo (Potter, Wyble, Hagmann, $\&$ McCourt, 2014).

$2^{\circ}$. La transformación del espacio del aula para potenciar el pensamiento divergente se ha reflejado en una mayor creatividad aumentando hasta un $25 \%$ el numero de soluciones encontradas a los problemas planteados, con relación a otros cursos.

Respecto al análisis cuantitativo realizado de los test llevados a cabo por los alumnos al concluir las materias, los resultados más significativos fueron:

$1^{\circ}$. El $100 \%$ de los alumnos indicaron que estaban totalmente de acuerdo con que:

- Transformar el espacio del aula (modificar la distribución del mobiliario, utilizar las paredes, etc) contribuye al desarrollo de la creatividad.

- Transformar el espacio del aula (modificar la distribución del mobiliario, utilizar las paredes, etc) favorece el trabajo en equipo.

$2^{\circ}$. El $80 \%$ de los alumnos indicaron que estaban totalmente de acuerdo con que:

- Diseñar y dibujar en grupo, contribuye a la creatividad y a encontrar soluciones innovadoras. 


\section{Conclusiones}

Esta experiencia nos ha mostrado que la implementación de la competencia Arts \& Design, ha ayudado de forma significativa a los alumnos a encontrar de forma ágil soluciones inteligentes a los problemas y retos planteados. En relación con la implementación de las tareas vinculadas a la competencia Arts \& Design, ha quedado patente que las aulas que actualmente hay disponibles en la universidad no favorecen el pensamiento divergente, y esto supone un gran esfuerzo tanto para los docentes como para el alumnado, a la hora de adaptar estos espacios en "rincones para soñar". Hay una necesidad imperativa en relación con la transformación de las aulas tradicionales, en espacio mas amigables, flexibles y creativos que faciliten la implementación del aprendizaje STEAM, la creatividad y el pensamiento innovador.

\section{Agradeciminetos}

Este trabajo se ha desarrollado en el marco del Equipo de Innovación y Calidad Educativa (EICE) Baños de realidad: hacia un aprendizaje visible, responsable e innovador. [EICE-AVRI-Realidad], coordinado por la profesora Virginia Santamarina-Campos, y del Proyectos de Innovación y Mejora Educativa "Aplicación de estrategias STEAM en las áreas de Ciencias Sociales y Artes mediante una metodología de Aprendizaje-Servicio", dirigido por la profesora María de-Miguel-Molina.

\section{Referencias}

Just, M., \& Ludtke, M. (2010). Watching the Human Brain Process Information - Nieman Reports. Retrieved from https://niemanreports.org/articles/watching-the-human-brain-process-information/

Potter, M. C., Wyble, B., Hagmann, C. E., \& McCourt, E. S. (2014). Detecting meaning in RSVP at 13 ms per picture. Attention, Perception, and Psychophysics, 76(2), 270-279. https://doi.org/10.3758/s13414-013-0605-Z

Punset, E. (2013). Redes- La mirada de Elsa: La creatividad - RTVE.es. Retrieved January 9, 2020, from http://www.rtve.es/alacarta/videos/redes/redes-mirada-elsa-

creatividad/1643868/\#aHR0cDovL3d3dy5ydHZ1LmVzL2FsYWNhenRhL2ludGVybm8vY29udGVudHRhYmxlLnN odG1sP2N0eD0xNzA1JmxvY2FsZT1lcyZwYWdlU216ZT0xNSZzZWN0aW9uRmlsdGVyPTU5NDMwJmFkdlN1Y XJjaE9wZW49ZmFsc2U=

Santamarina, V., Carabal, M. Á., de Miguel, M., \& de Miguel, B. (2018a). Collaborative Visual Language for the Development of Innovative Ideas. EDULEARN18 Proceedings, 1(July), 535-543. https://doi.org/10.21125/edulearn.2018.0216

Santamarina, V., Carabal, M. Á., de Miguel, M., \& de Miguel, B. (2018b). From the Idea To the Project. Collaborative Trees As an Analytical Tool. EDULEARN18 Proceedings, 1(July 2018), 517-528. https://doi.org/10.21125/edulearn.2018.0214

United Nations (2015). Sustainable Development Goals. Retrieved January 9, 2020, from https://www.un.org/sustainabledevelopment/

Universitat Politècnica de València (2013). Competencias Transversales: UPV. Retrieved January 9, 2020, from http:/www.upv.es/contenidos/COMPTRAN/ 